\title{
Evaluating the Distraction Potential of Connected Vehicles
}

\author{
Joonbum Lee \\ Department of Industrial and \\ Systems Engineering \\ University of Wisconsin-Madison \\ 1513 University Avenue \\ Madison, WI 53705 \\ 01-608-890-3637 \\ jlee299@wisc.edu
}

\author{
John D. Lee \\ Department of Industrial and \\ Systems Engineering \\ University of Wisconsin-Madison \\ 1513 University Avenue \\ Madison, WI 53705 \\ 01-608-890-3168 \\ jdlee@engr.wisc.edu
}

\author{
Dario D. Salvucci \\ Department of Computer Science \\ Drexel University \\ 3141 Chestnut St. \\ Philadelphia, PA 19104 \\ 01-215-895-2674 \\ salvucci@cs.drexel.edu
}

\begin{abstract}
Connected vehicles offer great potential for new sources of information, but may also introduce new sources of distraction. This paper compares three methods to quantify distraction, and focuses on one method: computational models of driver behavior. An integration of a saliency map and the Distract-R prototyping and evaluation system is proposed as a potential model. The saliency map captures the bottom-up influences of visual attention and this influence is integrated with top-down influences captured by Distract-R. The combined model will assess the effect of coordinating salient visual features and drivers' expectations, and in using both together, generate more robust predictions of performance.
\end{abstract}

\section{Categories and Subject Descriptors}

A.0 [GENERAL]: Miscellaneous: D.2.2 [SOFTWARE ENGINEERING]: Design Tools and Techniques-User interfaces

\section{General Terms}

Human Factors

\section{Keywords}

Connected vehicles, Distraction, ACT-R, Distract-R, Saliency map

\section{INTRODUCTION}

Driver distraction has become a critical issue for drivers and designers alike. Regan, Young, and Lee [1] defined driver distraction as "the diversion of attention away from activities critical for safe driving toward a competing activity" (p. 7). The results of driver distraction can be catastrophic [1]. For example, at least $25 \%$ of police-reported crashes were caused by driver inattention, and driver distraction caused over $50 \%$ of these crashes [2].

Sources of distraction are various. Others have classified distraction sources into six broad categories - "things brought into vehicle," "vehicle occupants," "moving object or animal in

Permission to make digital or hard copies of all or part of this work for personal or classroom use is granted without fee provided that copies are not made or distributed for profit or commercial advantage and that copies bear this notice and the full citation on the first page. To copy otherwise, or republish, to post on servers or to redistribute to lists, requires prior specific permission and/or a fee.

AutomotiveUI'12, October 17-19, Portsmouth, NH, USA.

Copyright (c) 2012 ACM 978-1-4503-1751-1/12/10 ... \$15.00 vehicle," "internalized activity," "external objects, events or activities," and "other source of distraction" (p. 253) [3]. Among these sources, we focus on interaction with "vehicle systems". More vehicle systems (e.g., in-vehicle navigation, collision warning system, traveler information, etc.) have been developed and introduced, and they represent new sources of distraction.

Distraction associated with a vehicle system is a particular concern for the next generation of "connected vehicles"-a concept that describes communication between vehicles and other vehicles $(\mathrm{V} 2 \mathrm{~V})$, roadside infrastructures (V2I), and beyondroadside infrastructures (V2X), enabled by wireless communication [4]. Connected vehicles offer great potential for new sources of information that can aid drivers. The main goals of the connected-vehicle system include enhancing safety, mobility, and sustainability [4]. However, the system may also introduce new sources of distraction that could undermine safety. The wealth of connected-vehicle information could lead designers to create complex displays that lead to long glances away from the road, or involve drivers in deciding among a complex array of alternate routes and destinations. Therefore, designers require a systematic approach to assessing and reducing distraction while providing drivers with the potential benefits of a connectedvehicle infrastructure.

To help assess and reduce distraction, recent work has explored ways to quantify and even predict distraction, with the ultimate goal of evaluating existing and new systems with respect to their potential to distract the driver. This work can be divided roughly into three broad areas. First, guidelines define distraction and specify how designers and manufacturers can develop less distracting devices [e.g., 5]. For instance, a set of Human Factors Guidelines from the Federal Highway Administration (FHWA) states that highly saturated blue should be avoided [6]. Such guidelines have the benefit of providing direct and precise answers to specific design questions [e.g., 7]. However, guidelines often take a reductionist approach that may not capture problems that emerge from an interaction of features, and may offer little to no guidance for new systems that deviate in significant ways from the concepts for which the guidelines were developed.

A second way to understand and alleviate distraction involves evaluating designs by collecting behavioral data from drivers. Data collection can be performed in controlled laboratory experiments [e.g., 8, 9] and in on-road field studies [e.g., 10, 11], and may use a variety of test environments ranging from desktop simulators and large-scale simulators [e.g., 8, 12] to instrumented vehicles [e.g., 9]. Behavioral driver data provide a holistic description of the phenomena being tested, and may thus offer a more comprehensive assessment of design features than possible through the use of guidelines. However, there are typically 
tradeoffs between the realism of the behaviors observed and the costs of collecting the data. For instance, a large-scale field study may provide the most comprehensive data but may be prohibitively expensive and time-consuming (not to mention may expose drivers to unacceptable safety risks), whereas a small-scale laboratory study can be done quickly but the resulting data may be less representative of actual driving situations. Especially for very novel systems, a rigorous behavioral study often cannot include all the possible design alternatives that need to be addressed.

A third way to evaluate the potential distraction assesses candidate designs with computational models of driver behavior. This approach relies on developing models that represent the cognitive processes and physical actions of a driver in various simulated situations. For example, the ACT-R driver model [13] was developed to simulate driver behavior in a highway-driving context, and was later extended to account for aspects of distraction from phone dialing [e.g., 14] and cognitive tasks [15]. Cognitive models have the benefit that a designer can simulate systematic tradeoffs among a large variety of design features, predicting potential behavior and distraction much more quickly and safely than with human drivers. However, the development of such models is time-consuming and knowledge-intensive, and ultimately limited to our understanding of human behavior as can be expressed in a computational framework.

Designers may have intuition for potential distraction from interfaces that they designed. This intuition might reflect the qualitative influence of color, contrast, or layout in a design. Computational models can confirm these intuitions, but can also indicate the precise quantitative effect of the design. In particular, repeated application of the models (e.g., Monte-Carlo simulation) can provide indication of the relative effect of each design parameter, their interactions and the likely variability associated with human performance with the design. In this way, computational models can confirm and extend designers' intuitions.

This paper focuses on this third approach to assessing distraction potential of in-vehicle information system - using computational cognitive models of driver behavior. Previous applications of ACT-R have demonstrated substantial promise of this approach through a rapid prototyping and evaluation tool called Distract- $R$ [16]. However, ACT-R and Distract-R have a limited ability to represent visual attention and design features that might lead to long glances away from the road. To extend ACT-R to better address the design challenges of connected vehicles, this paper describes how current models of driver visual attention and salience might extend Distract-R.

The following section places the ACT-R driver model in the context of other approaches to modeling driver behavior with a discussion of their strengths and limitations. The subsequent section describes a model of visual salience, fine-tuned for describing visual attention to vehicle displays - a critical concern for the design of connected vehicles. The last section describes how this visual salience model will be integrated into Distract-R, providing the system with a rigorous model of the factors that influence driver's visual attention

\section{MODELS OF DRIVER BEHAVIOR AND PERFORMANCE}

Over at least the past four decades, there have been numerous efforts to represent driver behavior as mathematical or computational models. A number of these models focused on control-theoretic representations of behavior [e.g., 17, 18, 19]. These models, however, are less amenable to treatments of driver distraction because they assume continuous attention to perceptual inputs that may not be directly accessible to the driver, such as speed and distance [20]. Other work has focused on the basic perceptual aspects of driving [e.g., 21, 22], but again with a representation that resembles continuous control rather than the intermittent, broken spans of attention central to distraction scenarios. Below, we review the efforts that relate most closely to our own, with a focus on predicting distraction from in-vehicle systems as is critical for connected vehicles.

\subsection{DPM: The Driver Performance Model}

The Driver Performance Model (DPM) is a computational model of driver's perception, cognition, and control processes to generate steering, braking, and acceleration inputs [23]. The model is a part of Interactive Highway Safety Design Model (IHSDM) that is a set of modules (e.g., geometrics, vehicle dynamic module, and driver model) to evaluate highway geometric design [24]. The DPM simulates interactions of driver and vehicle with roadway geometry, and provides prediction of the effects of highway geometry on driver behavior [25]. It consists of six computational functions (perception, speed decision, speed control, path decision, path control, and attention) and generates control actions (speed control and path control) in response to the situation [23]. The perception component receives the physical description of the situation from the input data (e.g., geometric component), and then translates the description to estimates of vehicle state and other relevant variables for the decision and control components [24]. The speed/path decision components compute desired speed/path profiles that reflect geometric features, and then the attention component modifies the information-processing components to reflect driver's mental capacity and attentional demand that are controlled by roadway geometry [24].

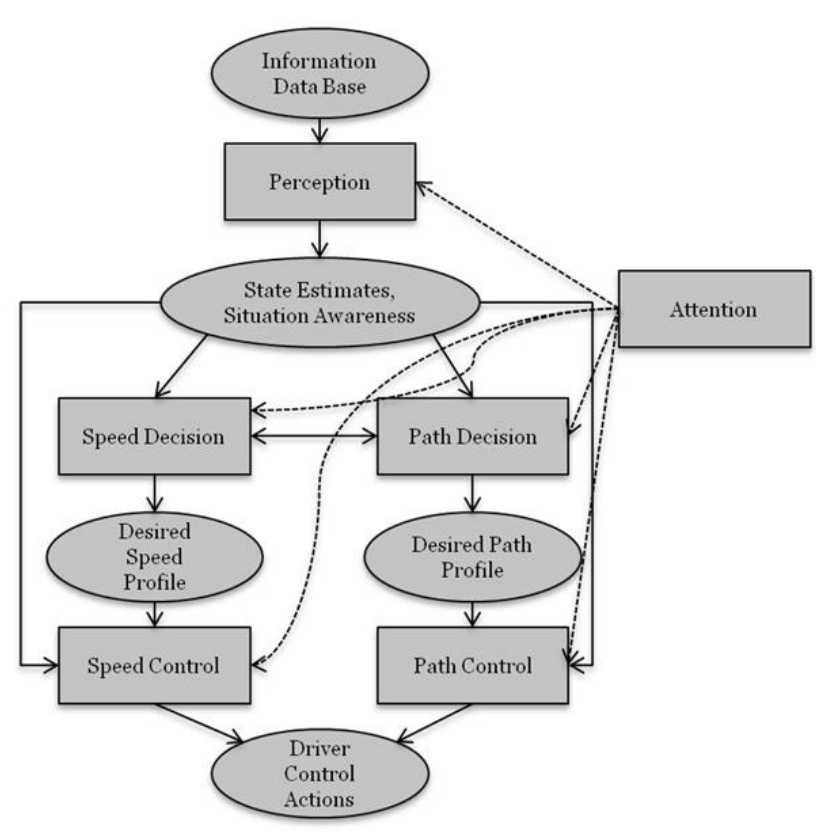

Figure 1: The Driver Performance Model (modified from Levison, Humm, Bittner, \& Simsek, 2001). 
The DPM can be used to evaluate impact of highway geometry, and simulate interaction among drivers, a vehicle, and roadway characteristics [23], but it does not deeply model human cognition, particular as it relates to how drivers share attention between the road and in-vehicle displays. Therefore, it has a limited capacity to evaluate the distraction potential of connectedvehicle information displays.

\subsection{The SEEV Model}

Wickens and colleagues [26] have reviewed models of visual information acquisition and identified four factors that affect visual attention. The four factors are physical "salience" of the scene, "effort" needed to move attention from previously fixated location, "expectancy" of the signal, and objective "value" of processing information. Based on these four factors, Wickens et al. [26] developed a descriptive model, known as the SEEV (Salience, Effort, Expectancy, and Value) model, to predict scanning and distribution of visual attention. In this model, the expectancy and value components account for top-down processing, whereas the salience and effort components account for bottom-up processing. The equation below shows that the four elements of this model combine in a linear fashion to predict probability of attending to an area [27]. The variables in the equation are described [27] as "S reflects the strength of salience, EF reflects the inhibitory strength of effort, EX or expectancy, reflects the collective forces of bandwidth (i.e., event frequency along a channel) and contextual cueing (i.e., event frequency given that an information cue has occurred), and $\mathrm{V}$ refers to the value" (p. 253).

$P(A)=s \boldsymbol{S}-e f \boldsymbol{E} F+(e x \boldsymbol{E} X+v \boldsymbol{V})$

(Note: Uppercase letters reflect the strength of each factor and lowercase letters represent coefficients for each factor.)

The SEEV model has been applied to study effects of in-vehicle tasks [28-31]. For example, Horrey and his colleagues [29] applied the SEEV model to drivers' visual attention while interacting with in-vehicle technologies (IVT). In this model, salience was not included because they defined salience as a property of specific events in a given area of interests (AOI) rather than physical properties of AOI. The model mainly predicts the probability of attending to a specific area [e.g.,'display' vs. 'outside world': 29], but does not generate predicted performance measurements (e.g., lane deviation or speed deviation in driving).

Recently, Steelman et al. [32] modified the SEEV model by integrating Walther and Koch's [33] salience model. However, there are limitations in applying this model to support design and to evaluate in-vehicle displays. For example, the modeler has to assign a "pertinence score" to each factor based on the usefulness for a given task condition/phase (e.g., in the study, two of authors independently assigned pertinence scores for each condition, and the AOIs' value and expectancy were estimated by a subject matter expert), Another limit of the model is that it cannot predict switching between tasks, whereas a critical aspect of distraction is the potential of a highly salient object might lead to long glances away from the road.

Our proposed model incorporates a model of task switching known as threaded cognition [14] and can predict measures of driving performance in addition to measures of visual attention such as dwell time to a visual area.

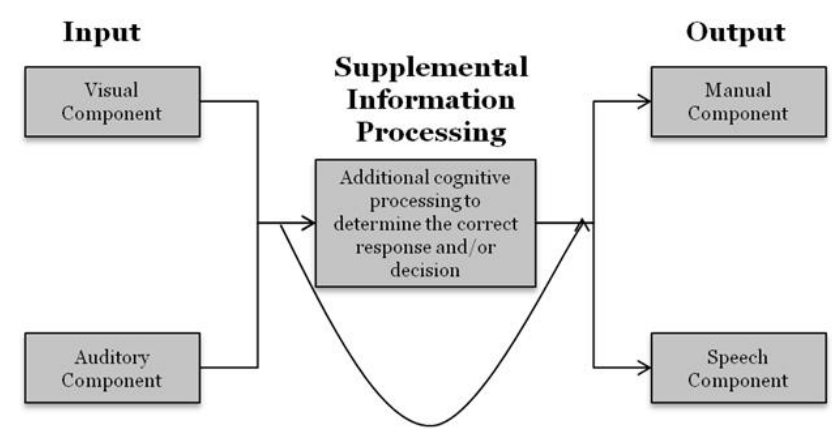

Figure 2: The behavioral model in DEMAnD (modified from Hankey, Dingus, Hanowski, \& Wierwille, 2000).

\subsection{IVIS DEMAnD}

The In-Vehicle Information System (IVIS) Design Evaluation and Model of Attentional Demand (DEMAnD) was developed to help designers of IVIS to evaluate attentional resources demanded by interactions with IVISs, and estimates the impact of these interactions on driving performance [34]. DEMAnD is not a computational model of driving per se, but rather a set of lookup tables and regression equations that combine task demands. The underlying theoretical assumption of DEMAnD is that drivers act with limited attentional resources and that resources demanded by a secondary task (e.g., interacting with IVISs) draw away from resources available for primary task (e.g., driving) and lead to decreased driving performance [34]. The behavioral model consists of five driver resources. The model uses various variables to measure demands, such as estimated single glance time to a display (in the visual resource), estimated number of message presentations (in the auditory resource), hand-at-task time (in the manual resource), and estimated number of command attempts (in the speech resource).

Several field studies were conducted to develop DEMAnD. Data from these studies and preexisting real-world data on driver-task measurement were fit with regression equations to produce a task library that includes 198 tasks (e.g., "adjust temperature") [34]. Users can specify characteristics of tasks (e.g., frequency of use, roadway complexity, and so on), driver population, driving environment, and other modifiers relevant to designs (e.g., display density, character height, and so on), and DEMAnD provides a hypothetical workload level and a summary of driving task performance [34]. One limitation of DEMAnD is that it does not provide specific measurements of performance such as lateralposition and speed deviation, and another one is that the model was not validated. Moreover, the model does not incorporate a detailed process model of driver behavior that would enable designers to assess a wide range of tasks and interface features. In many ways DEMAnD is more of an integration of design guidelines rather than a model of driver performance.

\subsection{Distract-R and ACT-R}

ACT-R [35] is a cognitive architecture that simulates human cognition. As a cognitive architecture, ACT-R aims to incorporate both the abilities and limitations inherent in human problem solving and response selection, including those involving memory, procedural skills, learning, and perceptual-motor behavior. With ACT-R as a base, the ACT-R driver model [13] was developed to model driver behavior in general, with an eye 


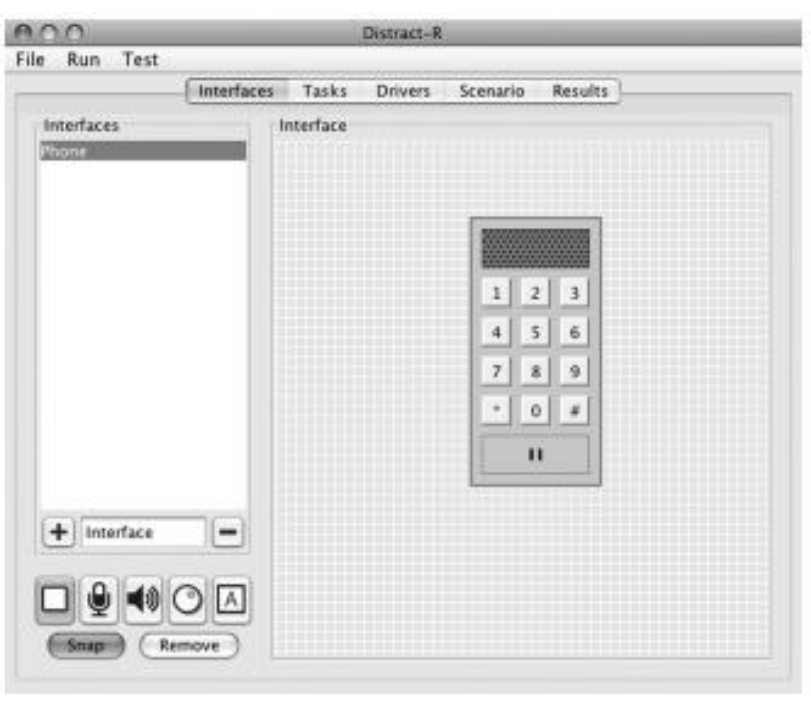

Figure 3: Distract-R screen image.

for modeling driver distraction as well. The driver model has been validated with respect to curve negotiation and lane-changing behavior in a highway context, as well as to steering and braking performance under distraction [see 14, 36, 37].

Although the ACT-R driver model can be used to predict driver distraction, the formal computational model requires experience with the ACT-R modeling language and programming the driving environment. To remedy this difficulty, Distract-R [16] was developed to provide a rapid prototyping and evaluation environment for the driver model; in essence, a user can benefit from the predictions of the core model, but the model itself is largely "under the hood," freeing the user to specify design alternatives and scenarios pertinent to their interests. Distract-R has been shown to generate reasonable predictions of distracted behavior for a few in-vehicle designs [16]. However, Distract-R and ACT-R are currently limited with respect to their account of visual attention: Although they can account for the timing of visual attention shifts and eye movements between visual objects [38], they rely on the human modeler to explicitly state how attention is directed from one place to the next. It would be more useful if the driver model to itself determine its path of visual attention, as driven by cues such as visual salience.

\section{MODEL OF VISUAL SALIENCE FOR ASSESSING IN-VEHICLE SYSTEMS}

The saliency map is a promising approach to assess how features of a scene influence visual attention. The saliency map represents visual saliency of a scene by modeling the factors that attract attention [39], and one advantage of the saliency map is that it captures the influence of visual feature contrast, along with local absolute feature strength [40]. Koch and Ullman [39] introduced the concept of a saliency map to predict preattentive selection by encoding the saliency of the objects in the visual environment. Walther and Koch's model [33] has been validated to predict sequential attention to objects in a complex scene by using three low-level visual features - color, intensity, and orientation - that may attract/distract drivers' visual attention.

The salience map builds on a series of computations that reflect the neurological processes associated with visual attention. The input image is sub-sampled and filtered by a Gaussian Pyramid that is widely used in image processing. Each pyramid level is decomposed into feature channels based on three low-level features (i.e., color, intensity, and orientation). Color is derived from red, green, blue, and yellow RGB values. Intensity is computed using an averaging function of the color features. Orientation $\left(0^{\circ}, 45^{\circ}, 90^{\circ}\right.$, and $\left.135^{\circ}\right)$ is computed by applying a Gabor filter (a linear filter for edge detection) to the images in the intensity pyramid. These features are summed by center-surround combinations and normalized. The resulting feature maps are combined into conspicuity maps - one for color, one for intensity, and one for orientation. Finally, the conspicuity maps are combined into a single saliency map that represents saliency as a scalar quantity at every location in the scene. In the saliency map, the location of the focus of attention is estimated using a WinnerTake-All (WTA) neural network that selects the most active location and suppresses the other locations.

Extended saliency maps [e.g., 41] have included motion and flicker features. The motion feature can be computed from spatially shifted differences in orientation between the current and previous frames. Similarly, flicker can be computed by calculating absolute differences in intensity between the current and previous frames [42].

The saliency map computes the influence of low-level visual features of a display that attract driver's attention, which can be the features that might draw driver's attention away from the area of display that contains the information of interest to the driver. Predicting the salience of display features can be central to estimating distraction potential, because it determines how likely an object is to attract or distract drivers' visual attention, and consequently, how many fixations might be required to find the desired information. If important information is highly salient relative to the background, it will be detected easily with a few fixations. However, misplaced salience-situations in which highly salient display features do not correspond to drivers' information needs - might lead to many fixations and long glances away from the road. The saliency map can identify instances of misplaced salience, which could help designers reduce the distraction potential of connected-vehicle displays.

The following section describes how the saliency model captures the bottom-up influences of visual attention and how this influence can be integrated with top-down influences (provided by ACT-R or another framework) to predict potential distraction and guide design.

\section{DISTRACTION POTENTIAL MODEL}

Both top-down, goal-driven (i.e., endogenous) and bottom-up, stimulus-driven (i.e., exogenous) processes govern visual attention [43]. Expectation, beliefs, and general knowledge of tasks provide a top-down influence on visual attention [44], and users of the connected vehicles also may have goal-driven input (e.g., spatial expectation for specific items in a display). Features of the display, such as the bright colorful elements of album art, might provide a bottom-up influence on visual attention. Therefore, an integrated model that accounts for both top-down and bottom-up mechanisms could provide a powerful way to describe the distribution of drivers' visual attention and predict potential distraction. Such a model must account for symbolic representations (such as goal and expectation) along with a continuous field of activation (such as the salience of visual stimuli). Figure 4 shows a conceptual representation of how Distract-R and the saliency map complement each other in guiding visual attention. Distract-R considers drivers' goal-driven, top-down processes and the saliency map considers drivers' 


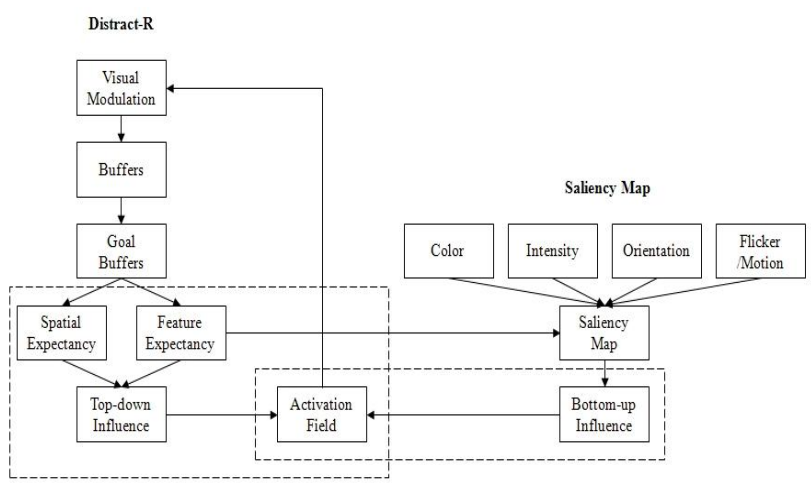

Figure 4: The proposed model to assess distraction, which integrates ACT-R and the saliency map. (Note: The dotted boxes represent where development will be done and other regions represent the current state of each model).

stimulus-driven, bottom-up process in visual attention. Figure 4 shows the top-down influence of Distract-R combining with the bottom-up influence of the saliency map through an activation field. The activation field links the symbolic representation from Distract- $R$ to the spatial representation of the saliency map to specify the most likely location of glances.

This model complements existing design guidelines by addressing important design issues that have not been fully addressed by those guidelines: 1) salience mapping (e.g., matching information importance to salience), 2) spatial consistency (e.g., consistent location of items on a display), and 3) feature consistency (e.g., consistent pairing of features to functions, such as the color red consistently representing a signal to stop).

For the technical integration of two models, Distract-R is currently developed to implement an ability to embed an interface image and to add hot spots on this image. This implementation allows designers to draft and test interface designs. The saliency map is modified to take input from Distract-R, and to return most salient item's coordinates with estimated searching time for the item. The revised saliency map code has two modes - a free viewing mode and a target searching mode - and the target searching mode requires target coordinates (x,y) as input and models successive visual scanning. The components of the model are coded in different programming languages, so we also modified Java API to communicate between Distract-R (in Java) and the saliency map (in Matlab).

\subsection{Model Application: Salience Mapping}

Consider a situation in which a GPS route guidance system requires a driver to decide between two routing options. If the driver is familiar with the device, and already knows the location of desired information, the driver's expectation guides visual attention to that location. However, if the driver does not have any prior knowledge and expectation, low-level visual features will guide attention. In this case, if important contents (e.g., required information to decide routing options) are highlighted and easy to find among other irrelevant contents (e.g., radio channels or advertisements), glance durations to the device will be shorter than when the irrelevant contents are highlighted with salient colors.

For example, the top image in Figure 5 is captured from Ford's "MyFord" interface. The saliency map can calculate saliency values for all location in the interface (The SaliencyToolbox [33]
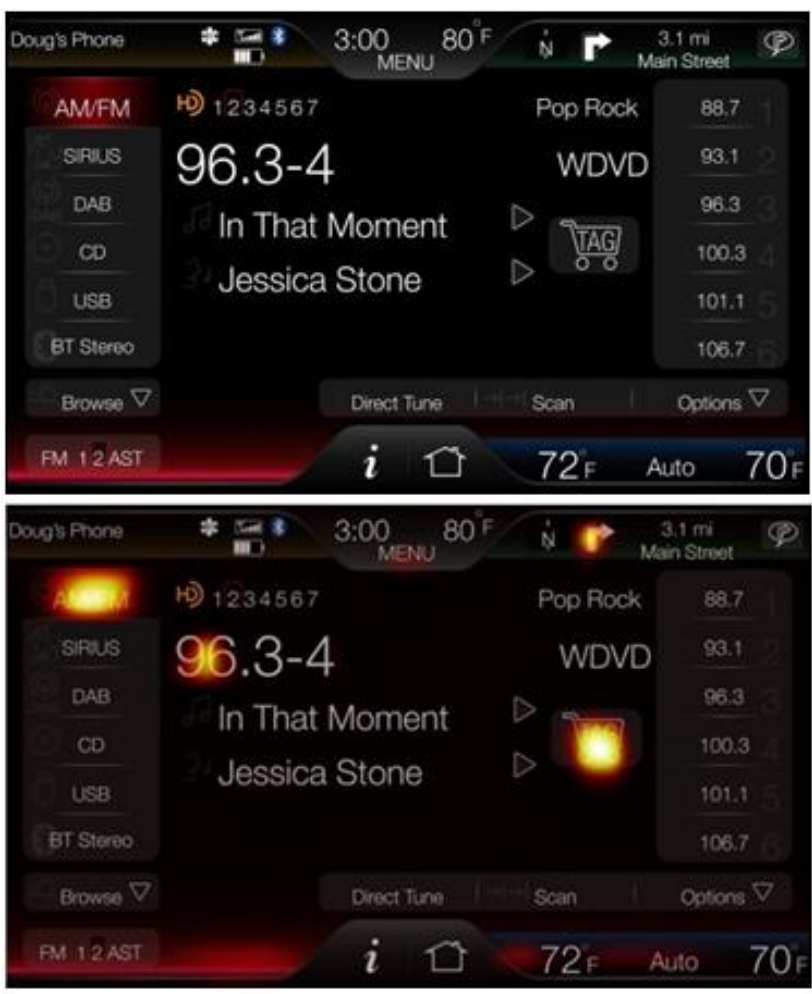

Figure 5: MyFord screen (top) and overlaid saliency map (bottom).
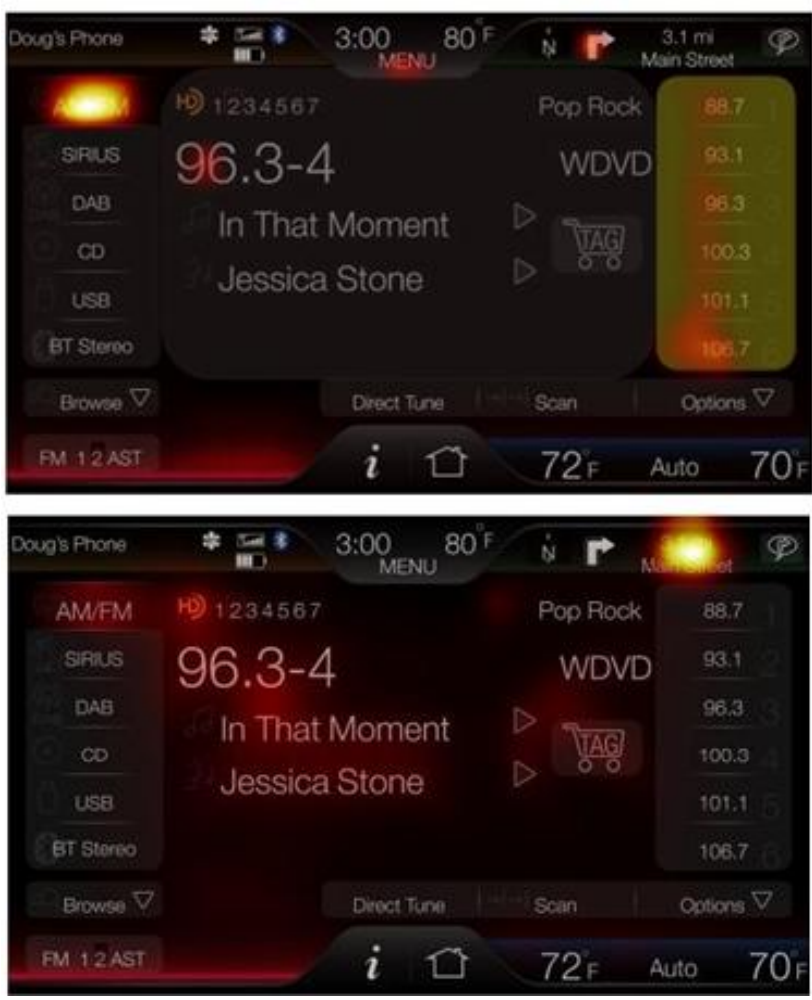

Figure 6: Overlaid saliency map after changing background color (top) and overlaid saliency map after implementation of flicker for turn information (bottom). 

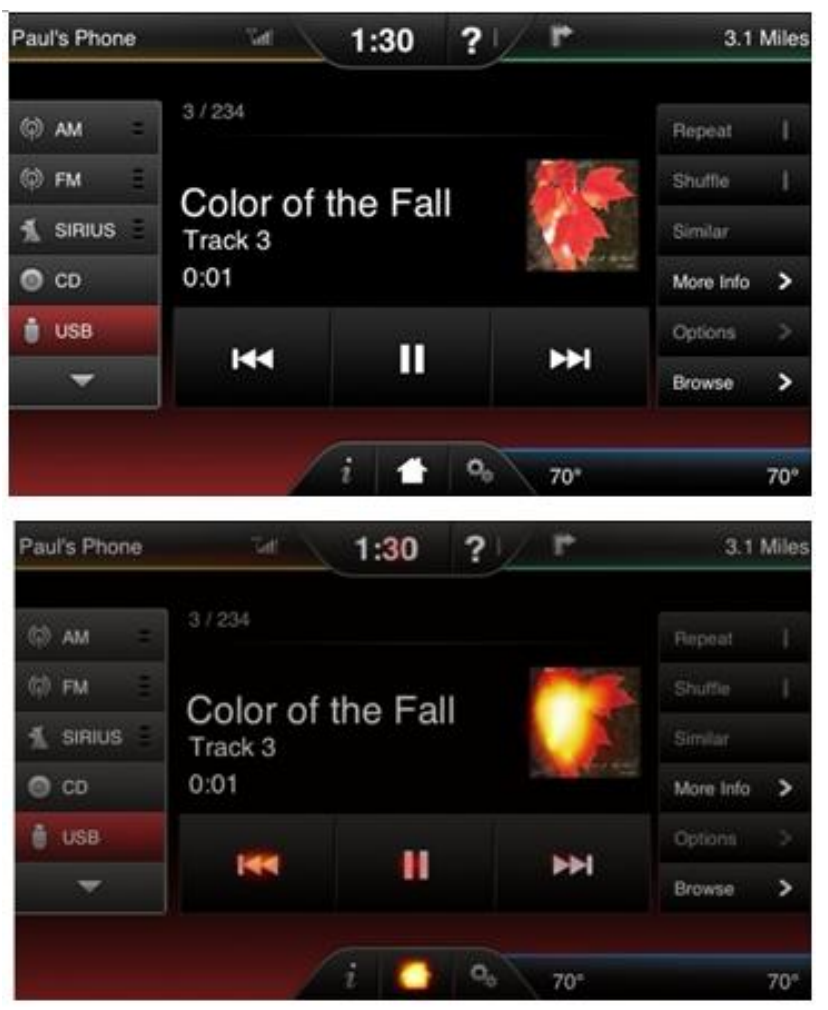

Figure 7: MyFord screen (top) and Overlaid saliency map (bottom).

and the Graph-Based Visual Saliency toolbox [41] are used to implement the saliency maps.) Based on the saliency map for the interface (the bottom image in Figure 5), visual features (i.e., bottom-up influences) may lead visual attention to the 'AM/FM' button that is highlighted with red color on the left side, white texts area that shows radio channel information on the center, or the shopping cart icon on the right side. The saliency map analysis could provide prediction for stimulus-driven attention, and could test an effect of changing design features. For example, if designers want to decrease salience effects on the center, decreasing contrast between background and text can be an alternative. Likewise, if designers want to lead attention to the channel box on the right side, a use of color for the area can be an alternative (e.g., we colored the right side of the MyFord screen to increase salience value for the area, and these effects are tested in the top image in Figure 6). A use of flicker can be another option. The bottom image in Figure 6 represents the flicker feature for a next turn information area (this information is located on the right-top corner). An additional image masking the next turn information with black color was used to create difference in intensity from the original image. Continuous switching between these two image frames implemented flicker, and this implementation increased visual salience of the specific area.

These examples show how design features could affect distraction potential. Consider a situation in which a driver needs to check information for the next turn, using the interface in Figure 5. In this case, the turn information that is relevant to a driving task does not have visual features that may attract driver attention. If the driver has no previous experience with the interface or does not know the location of the information, glance durations to the device will be longer than when the relevant contents are highlighted with salient features. Moreover, if there is misplaced salience on the interface (e.g., album art in Figure 7), the duration and number of glances tend to be increased, and distraction potential may increase. This potential effect of misplaced salience is also mentioned in National Highway Traffic Safety Administration (NHTSA)'s distraction guidelines for in-vehicles devices [45] by regulating a use of visual images, and the proposed model could complement this issue.

\subsection{Model Application: Spatial and Feature Expectation}

We propose an integrated model that includes bottom-up attention from the saliency map to form the activation field and top-down attention from Distract-R (see Figure 4). Drivers may have expectations, and these expectations can be primed with a specific goal that is passed from a goal buffer in Distract-R. The goal buffer, which stores the current goal, will pass a goal to perform a specific task on the connected-vehicle interface. For example, drivers may have spatial expectations for specific items or may expect specific features (such as color and shape for the items) for the target items. This type of expectation input can be converted to an augmented map format (for example, the spatial expectation can be implemented by increasing activation value for expected area of the interface, and the feature expectation can be implemented by differentially weighting the features), and combined with stimulus-driven attention to be processed by the saliency map. The final activation field including the most activated location (i.e., the "winning" location) is returned to a visual module in Distract- $\mathrm{R}$ to guide a path of visual attention until it finds the target item of the task.

Although we plan to implement memory modules in Distract-R and to integrate with the saliency map, the current version of Distract-R does not support modules to model drivers' expectation. Figure 8 shows projected examples of potential activation fields that combine top-down attention (e.g., spatial expectation) and bottom-up attention (e.g., visual features). Examples of activation fields in Figure 8 were created to show expected integration between those two attentional processes. Oliva and Torralba [46] implemented expectation of target location in terms of the likelihood of finding a local feature on a bottom-up based saliency map. The proposed model will apply a similar approach to implement the activation field, but the model will define drivers' expectation based on input from the cognitive architecture.

Through the implementation of the spatial expectation, the proposed model could assess the benefit of spatial consistency in the design. Spatial consistency would prime the activation field by increasing the value of the area associated with the information of interest (see Figure 8). Consistent placement of information and learning provides top-down attentional guidance that can counteract the effect of misplaced salience.

The top-down influence from Distract- $\mathrm{R}$ regarding feature expectations can be implemented by modifying the weight given to specific features, based on the drivers' tasks, goals, and knowledge about the target features. Wolfe [47] described the topdown process as a process that primes the possessing of specific features such as color or orientation. Consequently, the differential weighting of target features (e.g., specific color) can represent the top-down influence of Distract-R. This makes it possible to model the benefit of consistent coding of interface features, such as the when a driver who is looking for music 

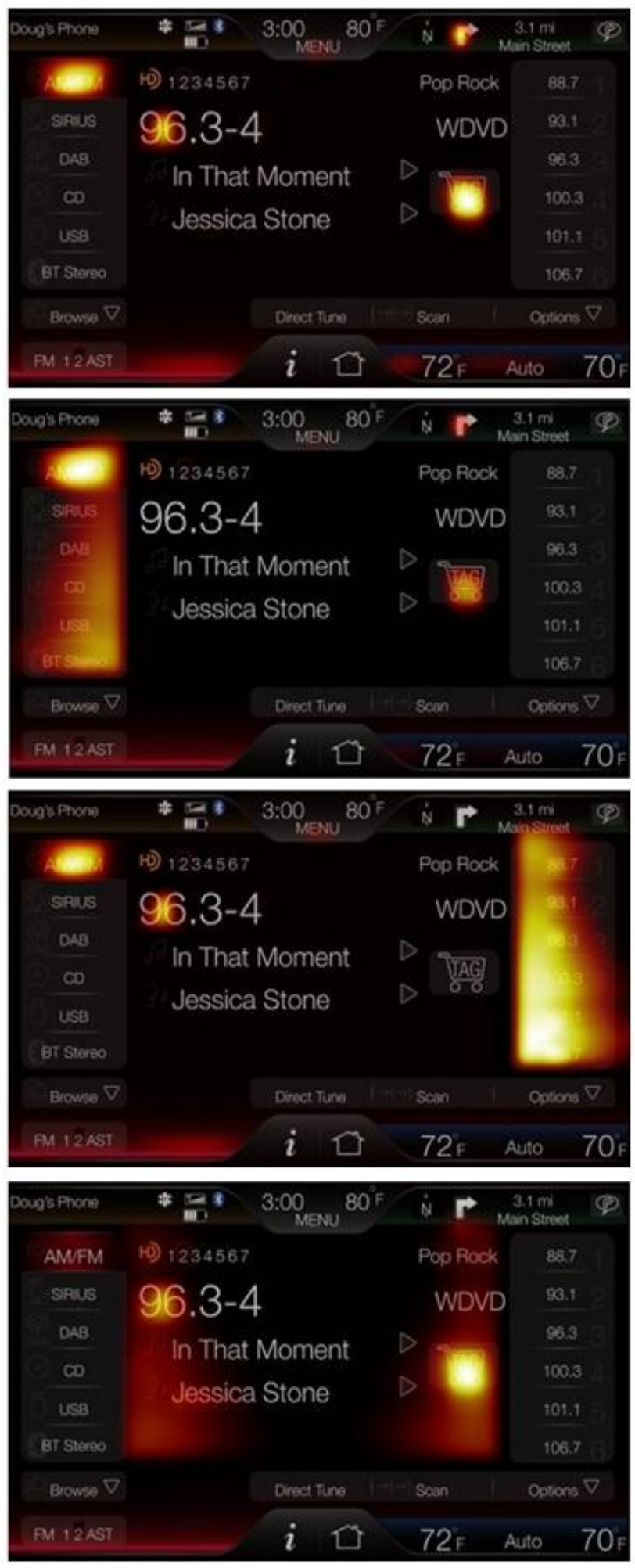

Figure 8: The MyFord interface with overlays: saliency map (1st image), activation field with spatial expectation for the left button box (2nd image), activation field with spatial expectation for the right button box (3rd image), and activation field with spatial expectation for the center $\left(4^{\text {th }}\right.$ image). information would be able to focus on the blue items if music were consistently coded with a blue color scheme.

A central issue concerning model development is the balance between the top-down influence from Distract- $\mathrm{R}$ and the bottomup influence of the saliency map. The activation field governs this relationship by combining the two inputs and guiding visual attention. Previous research has implemented the weighting of parameters by sequentially searching for the best value on a given validation data set [48]. A similar approach will be applied to balance top-down and bottom-up influences.

\section{CONCLUSION: POTENTIAL IMPACT}

The connected vehicle displays information from multiple sources, and driver distraction is recognized as a major issue in the connected-vehicles development [4]. As a first step to develop a computational model for evaluating the distraction potential of connected vehicles, previous models of driver behavior and performance were reviewed and we conclude that the future model needs abilities to 1) model threaded cognition to predict switching between tasks, 2) provide specific measurement of driving performance, and 3) account top-down and bottom-up mechanisms. The proposed model is expected to have those abilities based on the cognitive architecture, Distract-R, and the model of visual attention, the saliency map.

Distract-R will implement a time estimation module in ACT-R as a part of our model development, and this module will decide timing of visual attention shift. The ACT-R driver model already includes a stability threshold, and we approach the issue of dynamic visual switching by integrating vehicle stability, time estimation, and visual salience from displays. The process we designed consists of four steps that will be repeated while driving: 1) check vehicle stability, 2) set (or adjust) time threshold, 3 ) gaze to displays to find information needed (searching time will vary on interface features and will be estimated by the saliency map), and 4) gaze back to the road when duration of gaze away from the road exceeds the time threshold (or searching task is completed).

The final output of the proposed model will include driving performance variables (e.g., lateral deviation, speed deviation, and lane violation) and task completion time along with the ratio of gaze dwell time in various regions. Currently, only the visual influence from connected-vehicle displays is considered in our scope, but as mentioned previously, distraction caused by the roadway environment is also major issue. Modeling this influence will be one of the proposed model's future directions.

Consider again our sample concept of the connected-vehicle system with dynamic re-routing. When the system alerts the driver to a potential issue, it must now inform the driver about the reason for the alert, possible alternate routes, their associated travel times and costs, and so on. The visual display, as the most likely way to provide this information, becomes the critical bottleneck: a welldesigned display can afford the information in a quick convenient way, whereas a poorly designed display could confuse the driver and result in potentially hazardous distraction. For example, designers may use salient colors for more important information such as warnings and emergent information. This could promote faster search and reaction times, because low-level visual features draw attention, and drivers could have expectations for specific types of information after learning this color scheme. However, if the visual salience of the display runs counter to these expectations, the interface will need more time to guide the driver's attention to other information. 
As described before, the proposed model is expected to complement existing design guidelines. For example, Federal Highway Administration guidelines [6] suggest that highly saturated blue should be avoided, but it only considers the symbol color rather than the contrast between symbols and background color. In contrast, the proposed model could identify the effect of using specific colors or other visual features based on given backgrounds. As another example, guidelines published by the University of Michigan Transportation Research Institute [49] suggested using "discriminable colors." However, what constitutes a "discriminable color" depends on its interaction of other colors and display features. It is difficult for guidelines to indicate how combinations of interface features will influence driver attention to display, but such effects can be captured with the salience map process in the proposed model. Also, the commission of the European Communities [50] recommends that information with higher safety relevance should be given higher priority. Giving priority to safety relevance could also be tested with the proposed model as was demonstrated by the flicker feature for the small turn information area increased saliency value for its region and suppressed other location's salience in Figure 6.

In other situations, designers may spatially segregate information by its source or type, and drivers could have spatial expectations for specific target information. Given connected vehicles' multiple sources of information and overall information capacity, spatial segregation or color-coding will be important design features, and designers may need to consider influence of topdown mechanism along with bottom-up mechanism. The proposed model is expected to have the ability to examine the effect of coordinating salient visual features and drivers' expectations, and in using both together, generate robust predictions of performance. The commission of the European Communities [50] recommends to use well structured graphics on well positioned visual display, but without a computational model, designers may not be able to test the effect of spatial segregation or color coding, before collecting data with the interface or user tests.

NHTSA [45] lists tasks considered to be suitable for lock out on a per se basis, and one of the tasks is "Displaying photographic or graphical static visual images not related to driving. This would include album art and personal photos, among other things." (p. 69). Album art can result in misplaced salience; however, Gittins [51] pointed out that icons could carry more information within the same or less physical display space than other methods. Our model provides a tool for balancing the benefits of iconic album art with the potential for distraction associated with misplaced salience as shown in Figure 7. The proposed model is expected to have an ability to show how misplaced salience affects driving performance and how top-down process might compensate for misplaced salience.

The model proposed here can help to evaluate the display at this critical juncture, facilitating display design in such a way that salient features are readily available to drivers, and that attention is drawn directly to the most informative parts of the display.

\section{REFERENCES}

[1] Regan, M. A., Young, K. L. and Lee, J. D. 2009. Introduction. In Driver distraction : theory, effects, and mitigation (pp. 3-7). CRC Press, Boca Raton, FL.
[2] Stutts, J. C. and Hunter, W. W. 2003. Driver inattention, driver distraction and traffic crashes. Institute of Transportation Engineers Journal, 73, 34-45.

[3] Regan, M. A., Young, K. L., Lee, J. D. and Gordon, C. P. 2009. Sources of Driver Distraction. In Driver distraction : theory, effects, and mitigation (pp. 249-278). CRC Press, Boca Raton, FL.

[4] Kirk, B. 2011 Connected vehicles: an executive overview of the status and trends. Globis Consulting Inc, Ottawa, Canada.

[5] UN. ECE. Working Party on the Construction of Vehicles 1998. Consolidated Resolution on the Construction of Vehicles (R.E.3). UN, Geneva.

[6] Campbell, J. L., Carney, C., Kantowitz, B. H., TurnerFairbank Highway Research Center and Battelle Human Factors Transportation Center. 1998. Human factors design guidelines for Advanced Traveler Information Systems (ATIS) and Commercial Vehicle Operations (CVO). U.S. Dept. of Transportation, Federal Highway Administration, McLean, VA.

[7] Stevens, A., Cynk, S., Beesley, R. and TRL Limited. 2011. Revision of the checklist for the assessment of in-vehicle information systems. IHS, Bracknell, UK.

[8] Horberry, T., Anderson, J., Regan, M. A., Triggs, T. J. and Brown, J. 2006. Driver distraction: The effects of concurrent in-vehicle tasks, road environment complexity and age on driving performance. Accident Anal Prev, 38, 1, 185-191.

[9] Horrey, W. J. and Lesch, M. F. 2009. Driver-initiated distractions: examining strategic adaptation for in-vehicle task initiation. Accid Anal Prev, 41, 1, 115-122.

[10] Harbluk, J. L., Noy, Y. I., Trbovich, P. L. and Eizenman, M. 2007. An on-road assessment of cognitive distraction: Impacts on drivers' visual behavior and braking performance. Accident Anal Prev, 39, 2, 372-379.

[11] Stutts, J., Feaganes, J., Reinfurt, D., Rodgman, E., Hamlett, C., Gish, K. and Staplin, L. 2005. Driver's exposure to distractions in their natural driving environment. Accident Anal Prev, 37, 6, 1093-1101.

[12] Strayer, D. L. and Drews, F. A. 2004. Profiles in driver distraction: Effects of cell phone conversations on younger and older drivers. Hum. Factors, 46, 4, 640-649.

[13] Salvucci, D. D. 2006. Modeling driver behavior in a cognitive architecture. Hum. Factors, 48, 2, 362-380.

[14] Salvucci, D. D. and Taatgen, N. A. 2008. Threaded cognition: An integrated theory of concurrent multitasking. Psychological Review, 115, 1, 101-130.

[15] Salvucci, D. D. and Beltowska, J. 2008. Effects of Memory Rehearsal on Driver Performance: Experiment and Theoretical Account. Hum. Factors, 50, 5, 834-844.

[16] Salvucci, D. D. 2009. Rapid Prototyping and Evaluation of In-Vehicle Interfaces. Acm T Comput-Hum Int, 16, 2, 9:19:33.

[17] Brackstone, M. and McDonald, M. 1999. Car-following: a historical review. Transportation Research, 2, 181-196.

[18] Donges, E. 1978. A two-level model of driver steering behavior. Hum. Factors, 20, 691-707.

[19] Godthelp, H. 1986. Vehicle control during curve driving. Hum. Factors, 28, 211-221.

[20] Boer, E. R. 1999. Car following from the driver's perspective. Transportation Research-Part F, 2, 201-206.

[21] Fajen, B. R. and Warren, W. H. 2003. Behavioral dynamics of steering, obstacle avoidance, and route selection. $J$ Exp Psychol Human, 29, 2, 343-362. 
[22] Wilkie, R. and Wann, J. 2003. Controlling steering and judging heading: Retinal flow, visual direction, and extraretinal information. J Exp Psychol Human, 29, 2, 363378.

[23] Bittner, A. C., Simsek, O., Levison, W. H., Campbell, J. L. and Trb. 2002. On-road versus simulator data in driver model development - Driver performance model experience. Human Performance: Models, Intelligent Vehicle Initiative, Traveler Advisory and Information Systems: Safety and Human Performance, 1803, 38-44.

[24] Levison, W. H. 1998. Interactive highway safety design model - Issues related to driver modeling. Driver and Vehicle Modeling, 1631, 20-27.

[25] Levison, W. H., Humm, S. J., Bittner, A. C. and Simsek, O. 2001. Computational techniques used in the driver performance model of the interactive highway safety design model. Transp Res Record, 1779, 17-25.

[26] Wickens, C. D., Helleberg, J., Goh, J., Xu, X. and Horrey, B. 2001 Pilot task management: testing an attentional expected value model of visual scanning. University of Illinois, Aviation Research Lab, Savoy, IL.

[27] Wickens, C. D. and McCarley, J. S. 2008. Applied attention theory. CRC Press, Boca Raton, FL.

[28] Horrey, W. J. and Wickens, C. D. 2007. In-vehicle glance duration - Distributions, tails, and model of crash risk. Transp Res Record, 2018, 22-28.

[29] Horrey, W. J., Wickens, C. D. and Consalus, K. P. 2006. Modeling drivers' visual attention allocation while interacting with in-vehicle technologies. J Exp Psychol-Appl, 12, 2, 6778.

[30] Horrey, W. J. and Wickens, C. D. 2006. Examining the impact of cell phone conversations on driving using metaanalytic techniques. Hum. Factors, 48, 1, 196-205.

[31] Horrey, W. J. and Wickens, C. D. 2004. Driving and side task performance: The effects of display clutter, separation, and modality. Hum. Factors, 46, 4, 611-624.

[32] Steelman, K. S., McCarley, J. S. and Wickens, C. D. 2011. Modeling the Control of Attention in Visual Workspaces. Hum. Factors, 53, 2, 142-153.

[33] Walther, D. and Koch, C. 2006. Modeling attention to salient proto-objects. Neural Networks, 19, 9, 1395-1407.

[34] Hankey, J. M., Dingus, T. A., Hanowski, R. J. and Wierwille, W. W. 2000. The development of a design evaluation tool and model of attention demand. Retrieved from http://www.nrd.nhtsa.dot.gov/departments/nrd13/drivedistraction/PDF/8.PDFl, 2000.

[35] Anderson, J. R. 2007. How can the human mind occur in the physical universe? Oxford University Press, New York.

[36] Salvucci, D. D. 2001. Predicting the effects of in-car interface use on driver performance: an integrated model approach. Int J Hum-Comput St, 55, 85-107.
[37] Salvucci, D. D. 2005. A multitasking general executive for compound continuous tasks. Cognitive Sci, 29, 3, 457-492.

[38] Salvucci, D. D. 2001. An integrated model of eye movements and visual encoding. Cognitive Systems Research, 1, 201220.

[39] Koch, C. and Ullman, S. 1985. Shifts in Selective Visual Attention: Towards the Underlying Neural Circuitry. Human Neurobiology, 4, 219-227.

[40] Itti, L., \& Koch, C. 2001. Computational modelling of visual attention. Nature Reviews Neuroscience., 2, 3, 194-203.

[41] Harel, J., Koch, C. and Perona, P. 2006. Graph-Based Visual Saliency. Advances in Neural Information Processing Systems, 19, 545-552.

[42] Itti, L., Dhavale, N. and Pighin, F. 2003. Realistic Avatar Eye and Head Animation Using a Neurobiological Model of Visual Attention. In proceedings of the SPIE $48^{\text {th }}$ Annual International Symposium on Optical Science and Technology, San Diego, CA, 64-78.

[43] Theeuwes, J. 1994. Endogenous and Exogenous Control of Visual Selection. Perception, 23, 4, 429-440.

[44] Wright, R. D. and Ward, L. M. 2008. Orienting of attention. Oxford University Press, New York.

[45] National Highway Transportation Safety Administration. 2012. Visual-Manual NHTSA Driver Distraction Guidelines for In-Vehicle Electronic Devices. Federal Register, Washington, DC.

[46] Oliva, A. and Torralba, A. 2007. The role of context in object recognition. Trends in Cognitive Sciences, 11, 12, 520-527.

[47] Wolfe, J. M. 1994. Guided search 2.0 - a revised model of visual-search. Psychonomic Bulletin \& Review, 1, 2, 202238.

[48] Torralba, A., Oliva, A., Castelhano, M. S. and Henderson, J. M. 2006. Contextual guidance of eye movements and attention in real-world scenes: The role of global features in object search. Psychological Review, 113, 4, 766-786.

[49] Green, P., Levison, W. H., Paelke, G. and Serafin, C. 1995 Preliminary Human Factors Guidelines for Driver Information System. FHWA-RD-94-087, US Government Printing Office, Washington, DC.

[50] Commission of the European Communities. 2007. On safe and efficient in-vehicle information and communication systems: update of the European statement of principles on human machine interface. Official Jounal of the European communities, L31, 200-241.

[51] Gittins, D. 1986. Icon-Based Human Computer Interaction. Int J Man Mach Stud, 24, 6, 519-543. 